\title{
Adult Patients with Congenital Muscular Torticollis Treated with Bipolar Release : Report of 31 Cases
}

\author{
Gun Sang Lee, M.D., Myung Ki Lee, M.D., Woo Jae Kim, M.D., Ho Sang Kim, M.D., Jeong Ho Kim, M.D., Yun-Suk Kim, M.D. \\ Department of Neurosurgery, Maryknoll General Hospital, Busan, Korea
}

Objective : We assessed the surgical results of bipolar release in 31 adult patients with uncorrected congenital muscular torticollis (CMT) and more than 12 months of follow-up.

Methods : Thirty-one patients underwent a bipolar release of the sternocleidomastoid muscle (SCM) and were retrospectively analyzed. The mean follow-up period was 14.9 months (range, 12-30). The mean age at time of surgery was 30.3 years (range, 20-54). Patients were evaluated with a modified Lee's scoring system, cervicomandibular angle (CMA) measurement, and a global satisfaction rating scale using patient self-reporting.

Results : The modified Lee's scoring system indicated excellent results in 4 (12.9\%) patients, good in 18 (58.1\%), and fair in 9 (29.0\%) at the last follow-up after surgery. The improvements in neck movement and head tilt were statistically significant $(p<0.05)$. The preoperative mean CMA was $15.4^{\circ}$ (range, 5.4-29.0), which was reduced to a mean of CMA of $6.3^{\circ}$ (range, $\left.0-25\right)$ after surgery $(p<0.05$ ). The global satisfaction rating scale was $93.7 \%$ (range, 90-100). A transient sensory deficit on the ipsilateral lower ear lobe was noted in three cases. No significant permanent complications occurred.

Conclusion : Bipolar release of the SCM is a safe and reliable technique for the treatment of CMT in adults.

Key Words : Congenital · Torticollis · Adult · Sternocleidomastoid muscle · Tenotomy.

\section{INTRODUCTION}

Congenital muscular torticollis (CMT), caused by fibromastosis within the sternocleidomastoid muscle (SCM), refers to unilateral contracture of the SCM that restricts the range of motion at the neck and causes a head tilt toward the shortened $\mathrm{SCM}^{7,10)}$. Most infants with CMT are successfully managed with physical therapy ${ }^{4,5)}$. However, the torticollis, when uncorrected or resisted, sometimes results in tightness of the SCM, limited neck motion, head tilt, and craniofacial asymmetry. These cosmetic and functional problems can have an adverse impact on these patients in their adolescence and adulthood.

Several operation have been used to release the SCM : unipolar release, bipolar release, bipolar release with Z-plasty (Fig. 1), and endoscopic release $e^{3,8,10,12,16,24,25)}$. The results of sur-

- Received: March 31, 2016 • Revised: August 31, 2016 • Accepted: October 5, 2016

- Address for reprints : Myung Ki Lee, M.D.

Department of Neurosurgery, Maryknoll Medical Center, 121 Jungguro, Jung-gu, Busan 48972, Korea

Tel : +82-51-466-2456, Fax : +82-51-461-6164, E-mail : stereomk@naver.com

This is an Open Access article distributed under the terms of the Creative Commons Attribution Non-Commercial License (http://creativecommons.org/licenses/by-nc/4.0) which permits unrestricted non-commercial use, distribution, and reproduction in any medium, provided the original work is properly cited. 
gical release of the affected muscle for resistant cases are well recognized as good in infants, and even in older children ${ }^{2,10)}$. However, CMT is diagnosed and treated when a patient is a infancy or child, and it reported rarely in adult; consequently, reports on the efficacy of surgery for the uncorrected cases are limited and the best technique is still controversial ${ }^{9,15,18-20)}$. The aim of this study was to evaluated the results of bipolar release in 31 patients with CMT in adults with more than 12 months of follow-up.

\section{MATERIALS AND METHODS}

We retrospectively analyzed surgical outcomes of CMT in 31 adults whose bipolar release of the SCM was undertaken by a single neurosurgeon between August 2006 and February 2014. Each patient's wry neck had been noticed in infancy or early childhood by a parent. All patients met the following criteria : 1) congenital muscular torticollis with head tilt on the affected side and the presence of a tight band in the ipsilateral SCM, with absence of any previous surgery; 2) adult aged 20 years and older; and 3) no other congenital deformities in the neck determined by the magnetic resonance imag-
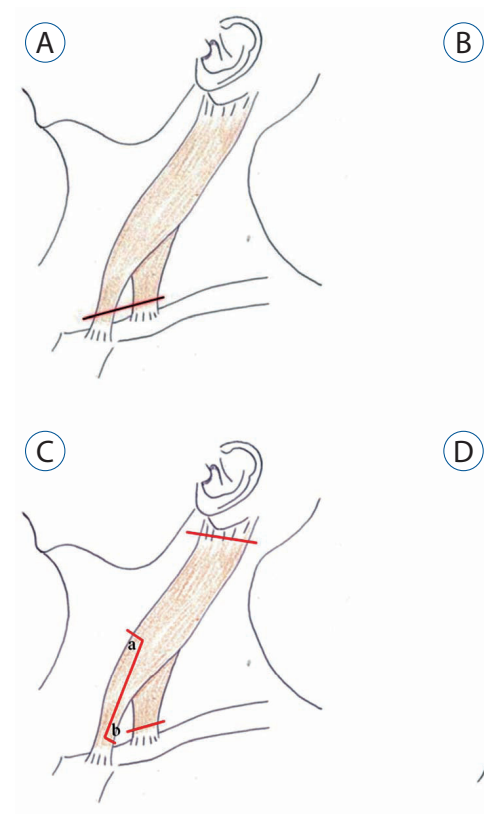

(B)

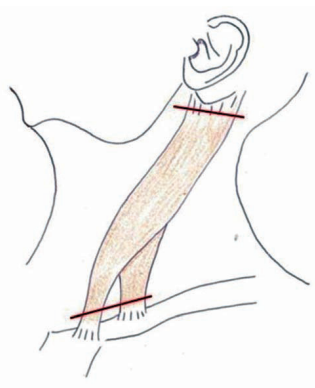

(D)

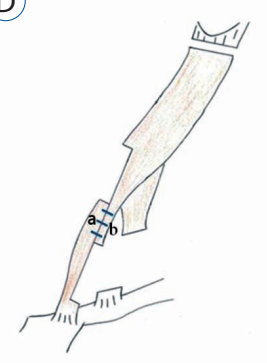

Fig. 1. Three popular operation for congenital muscular torticollis. A : Unipolar release. B : Bipolar release. C and D : Bipolar release with Z-plasty. ing or computed tomography. Thirty-one of 34 consecutive patients were followed up for more than 1 year after surgery; three patients were lost to follow-up. The clinical characteristics of the remaining 31 patients (15 males, 16 females) are summarized in Table 1. The right side was involved in 16 patients, and the left side in 15 . None of the patients presented with an additional congenital anomaly. The mean follow-up period was 14.9 months (range, 12-30). The mean age at the time of surgery was 30.3 years (range, 20-58).

All patients underwent a uniform procedure using bipolar release of the SCM. The procedure was conducted while the patient was under intravenous anesthesia and supine. The

Table 1. Clinical features of 31 adult patients with congenital muscular torticollis treated with bipolar release

\begin{tabular}{lc}
\hline Variable & Value \\
\hline Age (years) & $30.3 \pm 8.1$ (range, 20-58) \\
Follow-up (months) & $14.9 \pm 4.2$ (range, 12-30) \\
Sex (no.) & 15 \\
Male & 16 \\
Female & \\
Affected side (no.) & 16 \\
Right & 15 \\
Left & 15
\end{tabular}

Modified Lee's scoring system

Neck movement

Preoperative

$0.6 \pm 0.5$ (range, $0-1)$

Postoperative

$1.8 \pm 0.4$ (range, $1-2$ )

Head tilt

Preoperative

$1.4 \pm 0.8$ (range, $0-2$ )

Postoperative

$2.8 \pm 0.4$ (range,2-3)

Scar

$2.6 \pm 0.6$ (range,1-3)

Loss of column

$2.8 \pm 0.4$ (range,1-3)

Lateral band

$2.4 \pm 0.6$ (range, $1-3$ )

Postoperative outcome (no.)

Excellent

9 (29.0\%)

Good

18 (58.1\%)

Fair

$4(12.9 \%)$

Cervicomandibular angle $\left(^{\circ}\right)$

Preoperative

15.4 \pm 7.7 (range, 5.4-29.0)

Postoperative

$6.3 \pm 6.0$ (range, $0-25$ )

Satisfactory rating scale (\%)
$93.7 \pm 2.9$ (range, 90-100) 
patient's neck was extended with a sandbag, and then the patient's head was turned toward the shoulder on the opposite, uninvolved side. First, the distal portion of the SCM was released by making a skin incision 3 to $5 \mathrm{~cm}$ long on the medial end of the clavicle, with consideration given to palpable fibrous bands, and then deepening the incision to the tendons of the sternal and clavicular attachments of the SCM. The tight band and muscle tendon were dissected to pass a right angle instrument posterior to the tendons and were then resected by electrocautery near the insertion site to the clavicle. Next, the proximal portion of the SCM was released by making a short transverse skin incision $1.5 \mathrm{~cm}$ to $2 \mathrm{~cm}$ long behind the ear, just distal to the tip of the mastoid process. The SCM was then carefully dissected and released near the bone, while checking the nearby nerves and vessels. Finally, the wound was closed with Vicryl 4-0 to approximate the subcutaneous tissues, with meticulous repair of the platysma, The skin was then closed with Nylon 4-0.

A soft cervical collar was applied to maintain an upright position of the neck for at least 4 weeks after surgery. We recommended that each patient use the neck collar at home until 3 months after surgery. Each patient also received instructions for a manual stretching exercise and were directed to keep an upright position of the neck, while not wearing a brace, for at least 6 months. The intermittent stretching exercise was initiated 3 to 5 days after surgery.

Outcome assessment in this series was based on the modified Lee's scoring system ${ }^{19)}$ (Table 2), the cervicomandibular angle (CMA), and global satisfaction rating scale using patient self-reporting. Craniofacial asymmetry was noted in all patients; however, because craniofacial remodeling is not expected in adulthood, we used the modified Lee's scoring sys- tem that excluded the facial asymmetry section from Lee's scoring system $^{13)}$ for evaluation of the surgical outcome in our patients. The outcomes of this modified scoring system (Table 2 ), which included function (neck movement) and cosmesis (head tilt, operative scar, loss of column, and lateral band), were divided into the following four categories : an excellent result had a score of 14-15 points; good was 12-13 points; fair was 10-11 points; and poor was 9 or fewer points. The neck
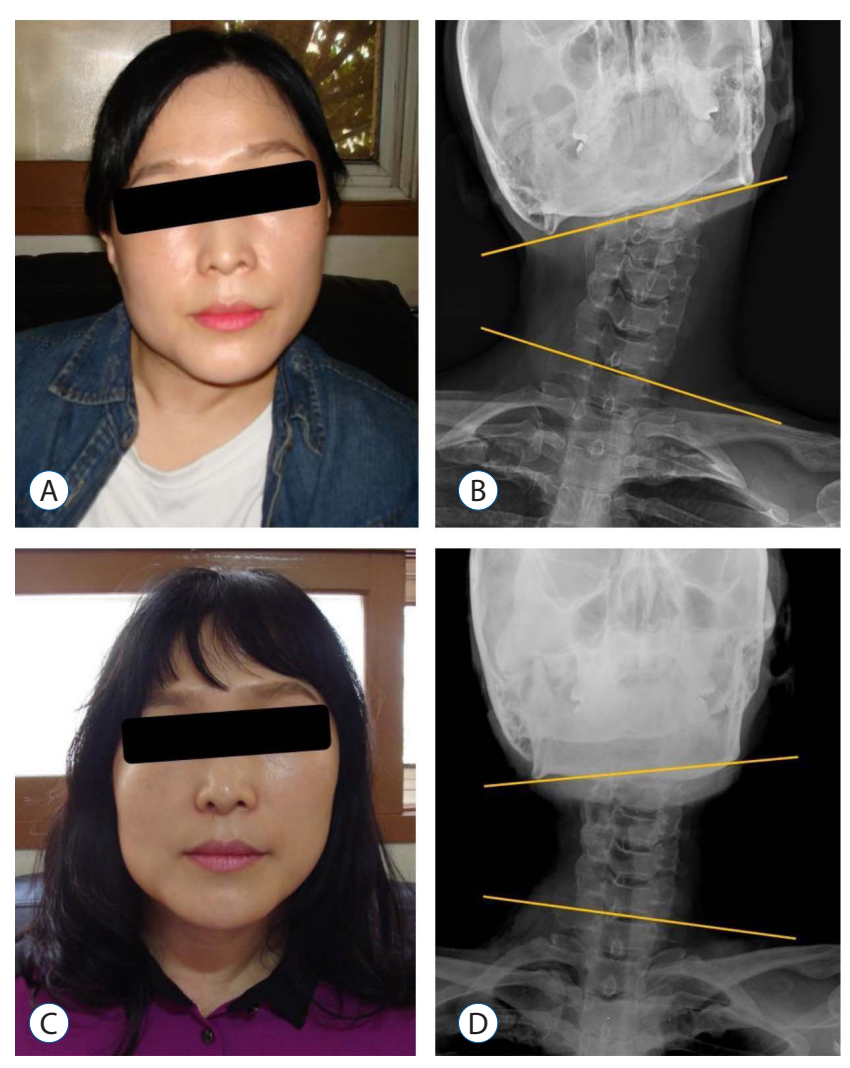

Fig. 2. A 42-year-old woman with uncorrected congenital muscular torticollis. Her head is tilted toward the right, with shoulder elevation and facial asymmetry. Preoperative photograph (A) and AP radiograph of the cervical spine (B). A postoperative photograph (C) and an AP radiograph of the cervical spine (D) of the same patient one year after bipolar release, showing improvement of head tilt and cervicomandibular angle.

Table 2. Modified Lee's scoring system for assessment of adult muscular torticollis

\begin{tabular}{lcllll}
\hline Points & Neck movement & Head tilt & Scar & Loss of column & Lateral band \\
\hline 3 & Full & None & Fine & None & None \\
2 & $<10^{\circ *}$ & Mild & Slight & Slight & Slight \\
1 & $10-25^{\circ *}$ & Moderate & Moderate & Obvious but & Obvious but \\
0 & & & & cosmetically acceptable & cosmetically acceptable \\
\hline
\end{tabular}

*Limitation 
movement of rotation and lateral bending were measured clinically by physical therapists with a goniometer equipped with an inclinometer (Clinical Range of Motion Instrument, Orthopedic Physical Therapy Products, Minnesota, USA) by comparing the contralateral side to the affected side. The head tilt, operative scar, loss of column, and lateral band were evaluated by clinical observation and a questionnaire. The degree of radiological head tilt was measured by determining the CMA, which is the angle between a line along the upper margin of the $\mathrm{C} 7$ vertebral body and a line connecting the lower margins of the mandibular angles (Fig. 2) ${ }^{22)}$.

Statistical analysis was performed using the Statistical Package for the Social Sciences (SPSS) software (ver. 12.0; SPSS Inc., Chicago, IL, USA). The statistical significance of the changes in neck movement deficit and CMA at last follow-up more than 1 year after surgery compared with the baseline was assessed using a paired $t$-test. A $p$ value $<0.05$ was considered statistically significant. Pearson correlation analysis was used to identify the relationship between clinical head tilt and radiological CMA.

\section{RESULTS}

The use of the modified Lee's scoring system gave excellent results for 4 (12.9\%) patients, good for 18 (58.1\%), and fair for $9(29.0 \%)$ at the last follow-up after surgery (Table 1). Preoperative limitation of movement in the neck was $10^{\circ}$ to $25^{\circ}$ in $18(58.0 \%)$ patients and above $25^{\circ}$ in 13 (42.0\%), which was improved to $10^{\circ}$ to $25^{\circ}$ in $7(22.6 \%)$ patients and below $10^{\circ}$ in 24 (77.4\%) after surgery. The preoperative mean score of neck movement was $0.6 \pm 0.5$ (range, $0-1$ ), which was improved to $1.8 \pm 0.4$ (range, $1-2$ ). Preoperative head tilt was mild in 8 (16.1\%) patients, moderate in $18(25.8 \%)$, and severe in 8 (58.1\%). Postoperative head tilt was rated as none in 25 $(80.6 \%)$ patients and mild in 3 (19.4\%). The preoperative mean score of head tilt of $1.4 \pm 0.8$ (range, $0-2$ ) was improved to a mean of head tilt of $2.8 \pm 0.4$ (range, $2-3$ ). These improvements in neck movement and head tilt were statistically significant $(p<0.05)$.
The appearance of scarring was fine in $22(71.0 \%)$ patients, slight in 7 (22.6\%), and moderate in 2 (6.4\%). Loss of column was rated as none in 24 (77.4\%) patients, slight in 7 (22.6\%), and obvious but cosmetically acceptable in 1 (3.2\%). The presence of a lateral band was rated as none in 13 (41.9\%) patients, slight in 17 (54.9\%), and obvious but cosmetically acceptable in $1(3.2 \%)$. The preoperative mean CMA was $15.4^{\circ}$ (range, 5.4-29.0), which was reduced to a mean of CMA of $6.3^{\circ}$ (range, $0-25)$ after surgery $(p<0.05)$. The correlation between clinical head tilt and radiological CMA before and after surgery reflected a fair degree of relationship, with a correlation coefficient of 0.450 . The global satisfaction rating score using patient self-reporting was 93.7\% (range, 90-100). A transient sensory deficit on the ipsilateral lower ear lobe was noted in three cases. The sensory problem dissipated nearly completely in three months. No wound infection, hematoma, or recurrence requiring surgery was encountered. No significant permanent complications occurred in any of the patients.

\section{DISCUSSION}

Controversy still remains regarding the timing and the method of surgical correction of CMT, and the best surgical technique in adult patients (Table 3 ). The age of the patient is a major factor in the treatment of CMT. Manual stretching

Table 3. Comparative result of surgical release of congenital muscular torticollis in adults

\begin{tabular}{|c|c|c|}
\hline Study & No. of patients & Mean age (yr) \\
\hline Omidi-Kashani et al. ${ }^{19)}$ & 14 & 21.9 (range, 18-32) \\
\hline Patwardhan et al. ${ }^{20)}$ & 12 & 24.0 (range, 17-31) \\
\hline Lim et al. ${ }^{15)}$ & 37 & 27.4 (range, 18-48) \\
\hline This study & 31 & 30.3 (range, 20-58) \\
\hline Procedure & & Result \\
\hline Bipolar & \multicolumn{2}{|c|}{ Excellent 7 , good 5 , fair 0 , poor $2^{*}$} \\
\hline \multirow[t]{2}{*}{ Bipolar with Z-plasty } & \multicolumn{2}{|c|}{ Excellent 8 , good 4 , fair 0 , poor $0^{\dagger}$} \\
\hline & \multicolumn{2}{|c|}{ Excellent 6, good 2, fair 4, poor $0^{*}$} \\
\hline Unipolar and bipolar & \multicolumn{2}{|c|}{ Excellent 21 , good 12 , fair 4 , poor $0^{\dagger}$} \\
\hline Bipolar & \multicolumn{2}{|c|}{ Excellent 9 , good 18 , fair 4 , poor $0^{*}$} \\
\hline
\end{tabular}

${ }^{*}$ Modified Lee's score, ${ }^{\dagger}$ Modified Cheng and Tang score 
exercise was effective in about $95 \%$ of patients who were first seen before the age of 1 year ${ }^{4,5)}$; however, CMT did not resolve spontaneously after 1 year of age $e^{6,10)}$. Facial bone asymmetry started to appear after 5 years of age ${ }^{23)}$. Any facial asymmetry could be prevented by surgery during early childhood, especially at 1 to 4 years of age, but the facial cosmetic result was unsatisfactory after surgery at over 6 years of age ${ }^{2,16)}$. In contrast, other observations ${ }^{22)}$ found no difference in recovery of craniofacial asymmetry between a younger group (1 to 4 years old) and an older group (5 to 16 years old). And Ling ${ }^{16)}$ reported that patients above the age of 5 years more commonly encountered late complications, like loss of the sternomastoid column, disfiguring scars, and the presence of lateral bands.

The most popular surgical treatment for CMT consists of unipolar (distal) or bipolar (proximal and distal) release of the SCM. The extent of tightness of the SCM determines the choice of unipolar or bipolar release. The former is viewed as appropriate for early childhood and mild deformity, the latter may be indicated for older patients and more severe deformity ${ }^{10,14)}$. Chen and $\mathrm{Ko}^{3)}$ recommended bipolar release as the treatment of choice for CMT in patients older than 6 years, whereas other authors ${ }^{17)}$ preferred to perform a distal release for patients older than 6 years. Lim et al. ${ }^{15)}$ reported that the site of release was determined intra-operatively, with bipolar release undertaken when manipulation could not give adequate correction after unipolar release, in order to reduce surgical morbidity in older children and adults. The rate of recurrence was almost 7\% after distal release, while low 2 to $2.9 \%$ rate of recurrence was after bipolar release ${ }^{4,25)}$. Ferkel et al. ${ }^{8)}$ described a modified bipolar release and Zplasty, which can preserve the normal contour of the muscle in the neck; the clavicular head was released completely while the sternal head was lengthened by Z-plasty. This approach is especially helpful to avoid sunken appearance of the distal end of the $\mathrm{SCM}^{10)}$. Patwardhan et al. ${ }^{20)}$ reported that bipolar release with Z-plasty in 12 adults with CMT preserved the neck line, but intraoperative estimation of the extent of lengthening was difficult. According to the Modified Lee scoring system, six patients had excellent results, two had good results and four had fair results. Some others ${ }^{3)}$ bipolar release with $\mathrm{Z}$-plasty indicate higher risk of more tethering or insufficient release of fibrous bands when compared to bipolar release without Z-plasty. In the earlier study of bipolar release without Z-plasty, with meticulous repair of the platysma and soft tissues, loss of the neck column was not cosmetic problem ${ }^{3,19)}$.

We found excellent results in $12.9 \%$ of patients, good results in $58.1 \%$, and fair in $29.0 \%$ in this report. Neither significant complications nor recurrences were encountered. These results are similar to those of a few authors who have reported benefits in the form of both cosmetic and functional improvements in the neck without serious complications after surgery in adult CMT patients ${ }^{9,18,19)}$. In the present study, a bipolar release without Z-plasty gave satisfactory results in the neck contour, with no loss of the lateral column of the muscle and with sufficient release of the lateral fibrous bands in most patients with meticulous repair of the platysma. Therefore, for adult CMT patients, we believe that a bipolar release without Z-plasty is less complicated and a simpler surgical treatment than bipolar release with Z-plasty.

Bipolar release of CMT in most adults treated in this study improved the head tilt and underlying secondary cervical spinal changes, similar to previous work ${ }^{9,15,18,19)}$. No disturbance was incurred by changing the deformed head position, even in a 58-year-old woman with severely uncorrected $\mathrm{CMT}^{11)}$ (Fig. 3). The adult patients adapted well to the new position of their necks. Their quality of life has improved dramatically since the operation, even in patients with irreversible craniofacial asymmetry. The high satisfaction rate above $90 \%$ obtained in this study is thought to reflect the good cosmetic benefit related to the head tilt and tight lateral band of the congenital disease through a relatively simple surgical release. The present result corresponds well with the earlier studies on children ${ }^{14)}$, which suggested that complete release of an abnormal tight fibrous band, even in adult patients with uncorrected CMT, is of critical importance.

Bipolar release of the SCM should be performed with caution to avoid injury the following major nerves and vessels : the facial and accessory nerves, internal and external jugular 

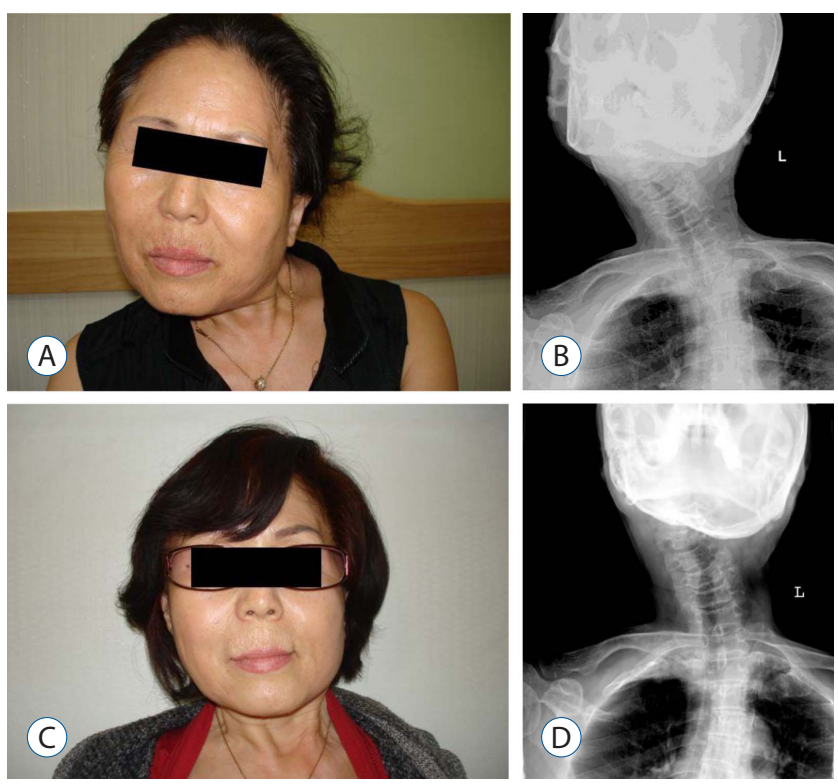

Fig. 3. A 58-year-old woman with uncorrected congenital muscular torticollis. Preoperative photograph (A) and AP radiograph of the cervical spine (B). 24 months after surgery, postoperative photograph (C) and an AP radiograph of the cervical spine (D) showing marked improvement of head tilt and lateral shift of the head.

veins, and carotid artery ${ }^{1,24,25)}$. In this study, post-operative pain and hypesthesia were major complaints. A transient sensory deficit was noted on the inferior part of ipsilateral ear lobe in three cases, but this dissipated nearly completely in 3 months. During proximal release, this was caused by injury to the posterior and intermediate branches of the great auricular nerve supplying the skin over the mastoid process and on the back of the auricle ${ }^{21)}$. Post-operative pain was markedly alleviated rapidly 3 days after surgery, resulting in a shortened hospital stay within 5 days after surgery and an earlier return to daily life activity in our patients.

Although a cast or halo-vest, cervical traction, and immobilization under overcorrected position were suggested for adult patients ${ }^{9,15,18)}$, we found that a more comfortable soft neck collar with early intermittent stretching exercise was sufficient to establish a neutral position of neck, in agreement with previous studies ${ }^{1,3,20)}$.

\section{CONCLUSION}

This study showed a statistically significant improvement of neck movement, head tilt, and lateral banding in most adult patients with CMT treated with bipolar release of the SCM. The patients were very satisfied with the functional and cosmetic results in the neck, even if their craniofacial asymmetry was unchanged, and none experienced any serious neurologic or spinal complications. Bipolar release of the SCM is therefore a safe and effective technique for the treatment of CMT in adults.

\section{References}

1. Amemiya M, Kikkawa I, Watanabe $H$, Hoshino $Y$ : Outcome of treatment for congenital torticollis : a study on ages for treatment, treatment methods, and postoperative therapy. Eur J Orthorp Surg Tramatol 19 : 303-307, 2009

2. Arslan $H$, Gündüz $S$, Subaşi $M$, Kesemenli $C$, Necmioğlu S : Frontal cephalometric analysis in the evaluation of facial asymmetry in torticollis, and outcomes of bipolar release in patients over 6 years of age. Arch Orthop Trauma Surg 122 : 489-493, 2002

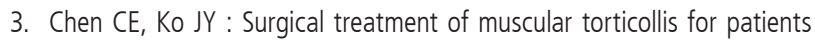
above 6 years of age. Arch Orthop Trauma Surg 120 : 149-151, 2000

4. Canale ST, Griffin DW, Hubbard CN : Congenital muscular torticollis. A long-term follow-up. J Bone Joint Surg Am 64 : 810-816, 1982

5. Cheng JC, Tang SP, Chen TM, Wing MW, Wong EM : The clinical presentation and outcome of treatment of congenital muscular torticollis in infants - a study of 1,086 cases. J Pediatr Surg 35 : 1091-1096, 2000

6. Coventry MB, Harris LE : Congenital muscular torticollis in infancy; some observations regarding treatment. J Bone Joint Surg Am 41 : 815-822, 1959

7. Do TT : Congenital muscular torticollis: current concepts and review of treatment. Curr Opin Pediatr 18 : 26-29, 2006

8. Ferkel RD, Westin GW, Dawson EG, Oppenheim WL : Muscular torticollis. A modified surgical approach. J Bone Joint Surg Am 65 : 894-900, 1983

9. Ippolito E, Tudisco C : Idiopathic muscular torticollis in adults. Results of open sternocleidomastoid tenotomy. Arch Orthop Trauma Surg 105 : 49-54, 1986

10. Kelly DM : Congenital anomalies of the trunk and upper extremity in Cannale ST, Beaty JH (eds) : Campbell's Operative Orthopaedics, ed 12. Philadelphia : Elsevier, 2013, Vol 2, pp1119-1132

11. Kim WJ, Lee MK, Lee GS, Kim HS, Kim JH, Kim YS : Congenital muscular torticollis of a woman aged 58 years satisfactorily treated with surgical release. J Kor Soc Ster Func Neurosurg 11 : 128-130, 2015

12. Lee TG, Rah DK, Kim YO : Endoscopic-assisted surgical correction for congenital muscular torticollis. J Craniofac Surg 23 : 1832-1834, 2012 
13. Lee EH, Kang YK, Bose $K$ : Surgical correction of muscular torticollis in the older child. J Pediatr Orthop 6 : 585-589, 1986

14. Lee IJ, Lim SY, Song HS, Park MC : Complete tight fibrous band release and resection in congenital muscular torticollis. J Plast Reconstr Aesthet Surg $63: 947-953,2010$

15. Lim KS, Shim JS, Lee YS : Is sternocleidomastoid muscle release effective in adults with neglected congenital muscular torticollis? Clin Orthop Relat Res 472 : 1271-1278, 2014

16. Ling CM : The influence of age on the results of open sternomastoid tenotomy in musculartorticollis. Clin Orthop Relat Res (116) : 142-148, 1976

17. Minamitani K, Inoue A, Okuno T : Results of surgical treatment of muscular torticollis for patients greater than 6 years of age. J Pediatr Orthop $10: 754-759,1990$

18. Oh I, Nowacek CJ : Surgical release of congenital torticollis in adults. Clin Orthop Relat Res (131) : 141-145, 1978

19. Omidi-Kashani F, Hasankhani EG, Sharifi R, Mazlumi $M$ : Is surgery recommended in adults with neglected congenital muscular torticollis? A prospective study. BMC Musculoskelet Disord 9 : 158, 2008
20. Patwardhan S, Shyam AK, Sancheti P, Arora P, Nagda T, Naik P : Adult presentation of congenital muscular torticollis: a series of 12 patients treated with a bipolar release of sternocleidomastoid and Z-lengthening. J Bone Joint Surg Br 93 : 828-832, 2011

21. Romans GJ : The peripheral nervous system in Romans GJ (eds) : Cunningham's textbook of anatomy, ed 12. Oxford: Oxford University Press, 1981, pp739-827

22. Shim JS, Jang HP : Operative treatment of congenital torticollis. J Bone Joint Surg Br 90 : 934-939, 2008

23. Yu CC, Wong FH, Lo LJ, Chen YR : Craniofacial deformity in patients with uncorrected congenital muscular torticollis : an assessment from three-dimensional computed tomography imaging. Plast Reconstr Surg $113: 24-33,2004$

24. Yuan B, Qu F, Zhao G, Wang J, Shen X, Liu Y : Arthroscopic surgical treatment for neglected congenital muscular torticollis in adults. J Craniofac Surg 26 : 512-515, 2015

25. Wirth CJ, Hagena FW, Wuelker N, Siebert WE : Biterminal tenotomy for the treatment of congenital muscular torticollis. Long-term results. J Bone Joint Surg Am 74 : 427-434, 1992 